\title{
Non-radiative processes in metal halide perovskite semiconductors probed by photoluminescence microscopy
}

\author{
Aboma Merdasa ${ }^{4}$, Marina Gerhard ${ }^{1}$, Boris Louis ${ }^{1,3}$, Jun $\mathrm{Li}^{1}$, Alexander Dobrovolsky ${ }^{1}$, \\ Yuxi Tian ${ }^{3}$, Johan Hofkens ${ }^{3}$, Rafael Camacho ${ }^{3}$, Eva Unger ${ }^{2}$, and Ivan G. Scheblykin ${ }^{1, *}$ \\ ${ }^{1}$ Chemical Physics and NanoLund, Lund University, PO Box 124, SE-22100, Lund, Sweden \\ ${ }^{2}$ School of Chem. \& Chem. Engineering, Nanjing University, 22 Hankou Rd, Nanjing 210023, China \\ ${ }^{3}$ Department of Chemistry, KU Leuven, Celestijenlaan 200F, B-3001 Leuven, Belgium \\ ${ }^{4}$ Helmholtz-Zentrum Berlin für Mater. und Energie GmbH, Kekulestraße 5, 12489 Berlin, Germany
}

\begin{abstract}
Organo metal halide perovskites are solution processed semiconductors that recently attracted a great attention. They possess a rather "soft" and (photo) chemically active solid structure allowing for ion migration and other mass diffusion processes. This is a likely reason why non-radiative recombination centres in these materials are activated and deactivated on relatively slow time-scales. This dynamics reveals as photoluminescence (PL) fluctuations (blinking) of individual microcrystals and local areas of films and allows for application of a broad range of single molecule spectroscopy methods including optical super-resolution. Studying PL blinking resolves properties of individual non-radiative centres and helps to unravel their chemical nature.
\end{abstract}

Non-radiative charge recombination in semiconductors sets a fundamental limitation to the efficiencies of light emitting devices and solar cells. Recently re-discovered organometal-halide perovskites (OMHP) have become the most popular and, in a way, mysterious optical semiconductors showing a great potential for industrial applications. It was quickly realized that spectroscopy methods able to monitor the fate of photogenerated charge carriers locally with high spatial resolution are of a high demand. [1] The resolution in space is needed because these solution-processed semiconductors possess highly inhomogeneous properties in terms of polycrystallinity, defect state distributions, formation of intermediates and degradation. Photoluminescence (PL) micro-spectroscopy is one of such methods. OMHPs possess phenomena of photo-brightening, defect curing, ion migration, and other light-induced photochemical processes which can be nicely monitored by PL micro-spectroscopy [1]. In several publications we have successfully used ideas coming from single molecule spectroscopy to study perovskite films and individual nanoand micro-crystals [2-4]. OMHP materials are highly dynamic in terms of local chemical structure leading to easily observable temporal fluctuations of PL yield of localized regions of perovskite films [5] or individual crystals [2-4]. PL flickering or blinking is the direct indication of switching processes in efficient non-radiative recombination centres.

Non-radiative recombination in semiconductors is a difficult process to comprehend in

\footnotetext{
*Corresponding author: ivan.scheblykin@ chemphys.lu.se
} 
detail $[6,7]$. It occurs due to the presence of intra-band defect states (traps) or complex nonradiative centres. The concentration of these quenchers and the charge trapping cross sections per one quencher are usually not known. Luckily OMHPs possess properties that allowed us to see and count each individual non-radiative channel in a small particle. These properties are crystal structure dynamics and mass (ion) diffusion, leading to transformation of the material at slow time scale of milliseconds, seconds and beyond. It turned out that these dynamics lead to activation/deactivation of individual non-radiative channels observed experimentally as PL blinking. The efficiency of just one channel can be very large, allowing for on/off switching of the PL of $500 \mathrm{~nm}$ sized $\mathrm{MAPbI}_{3}$ crystals and substantial fluctuations of the PL of 10 micrometer long $\mathrm{MAPbI}_{3}$ rods with a volume of about $1 \mu \mathrm{m}^{3}[2,4]$. It was suggested that such an efficient quencher (a super-trap or a "killing" centre) can be a donor-acceptor complex [2]. Monitoring of PL fluctuations allows us to obtain information about charge carrier and exciton diffusion, trapping and trap concentrations. Moreover, the switching allows for application of optical super resolution spectroscopy to localize trapping and emitting sites in space with nanometer accuracy $[2,8]$.

Single molecule fluorescence and its blinking gave birth to super-resolution optical microscopy (Nobel Prize 2014 in chemistry). Similarly, fluctuating activity of individual quenchers in OMHPs allowed us to get a mechanistic view beyond ensemble averaging on the non-radiative recombination in these semiconductors. We were able to resolve the temperature-dependent action of each defect state individually by observing $50 \mathrm{~nm}$ sized $\mathrm{MAPbI}_{3}$ crystals and studying the increase of their PL intensity upon cooling from $300 \mathrm{~K}$ to $77 \mathrm{~K}$. We suggest that the temperature activation of PL quenching is related to the potential barriers present in charge carrier diffusion and in charge trapping process. Contrary to some literature [9] we propose that the apparent activation energy of the PL intensity is not directly related to the exciton binding energy. Individual crystals showed highly diverse temperature dependencies of PL, which in many cases could not be approximated by the Arrhenius equation. Further studies are on the way towards Monte-Carlo simulations of the PL blinking dynamics and estimation of activation energies of the non-radiative centre formation and passivation and correlating these energies with the activation barriers of mass diffusion processes in perovskites including ion migration.

\section{References}

1. C. S. Ponseca, Y. Tian, V. Sundström, and I. G. Scheblykin, Nanotechnology 27, 82001 (2016)

2. A. Merdasa, Y. Tian, R. Camacho, A. Dobrovolsky, E. Debroye, E. L. Unger, J. Hofkens, V. Sundström, and I. G. Scheblykin, ACS Nano 11, 5391 (2017)

3. A. Dobrovolsky, A. Merdasa, E. L. Unger, A. Yartsev, and I. G. Scheblykin, Nat. Commun. 8, 34 (2017)

4. Y. Tian, A. Merdasa, M. Peter, M. Abdellah, K. Zheng, C. S. Ponseca, T. Pullerits, A. Yartsev, V. Sundström, and I. G. Scheblykin, Nano Lett. 15, 1603 (2015)

5. A. Halder, R. Chulliyil, A. S. Subbiah, T. Khan, S. Chattoraj, A. Chowdhury, and S. K. Sarkar, J. Phys. Chem. Lett. 6, 3483 (2015)

6. V. N. Abakumov, V. I. Perel, and I. N. Yassievich, Nonradiative Recombination in Semiconductors (North-Holland, Amsterdam, 1991)

7. S. D. Stranks, ACS Energy Lett. 2, 1515 (2017)

8. Y. Tian, A. Merdasa, E. Unger, M. Abdellah, K. Zheng, S. McKibbin, A. Mikkelsen, T. Pullerits, A. Yartsev, V. Sundström, and I. G. Scheblykin, J. Phys. Chem. Lett. 6, $4171(2015)$

9. T. J. Savenije, C. S. Ponseca, L. Kunneman, M. Abdellah, K. Zheng, Y. Tian, Q. Zhu, S. E. Canton, I. G. Scheblykin, T. Pullerits, A. Yartsev, and V. Sundström, J. Phys. Chem. Lett. 5, 2189 (2014) 